\title{
Whole-mount pathology of breast lumpectomy specimens improves detection of tumour margins and focality
}

\author{
Gina M. Clarke, Claire M. B. Holloway, Judit T. Zubovits, \\ Sharon Nofech-Mozes, Kela Liu, Mayan Murray, Dan Wang \\ and Martin J. Yaffe
}

Version Post-Print/Accepted Manuscript

Citation Clarke G.M., Holloway C.M., Zubovits J.T., Nofech-Mozes S., Liu K., (published version) Murray M., Wang D., Yaffe M.J., Whole-mount pathology of breast lumpectomy specimens improves detection of tumour margins and focality., Histopathology Volume 69, Issue 1, July 2016, Pages 35-44, doi: $10.1111 /$ his. 12912

\begin{abstract}
Publisher's Statement This is the peer reviewed version of the following article: Clarke G.M., Holloway C.M., Zubovits J.T., Nofech-Mozes S., Liu K., Murray M., Wang D., Yaffe M.J., Whole-mount pathology of breast lumpectomy specimens improves detection of tumour margins and focality., Histopathology Volume 69, Issue 1, July 2016, Pages 35-44, which has been published in final form at https://dx.doi.org/10.1111/his.12912. This article may be used for non-commercial purposes in accordance with Wiley Terms and Conditions for Self-Archiving.
\end{abstract} accepted manuscript) because you cannot access the published version, then cite the TSpace version in addition to the published version using the permanent URI (handle) found on the record page. 


\section{Whole-mount pathology of breast lumpectomy specimens improves detection of tumour margins and focality}

Short title: Whole-mount pathology: tumour margins and focality

Gina M Clarke, ${ }^{1}$ Claire M B Holloway, ${ }^{2,3}$ Judit T Zubovits, ${ }^{4,5}$ Sharon Nofech-Mozes, ${ }^{5,6}$ Kela Liu, ${ }^{1}$ Mayan Murray, ${ }^{1}$ Dan Wang ${ }^{1} \&$ Martin J Yaffe ${ }^{1,7}$

${ }^{1}$ Physical Sciences, Sunnybrook Research Institute, ${ }^{2}$ Department of Surgery, Sunnybrook Health Sciences Centre, ${ }^{3}$ Department of Surgery, Faculty of Medicine, University of Toronto, ${ }^{4}$ Department of Pathology, The Scarborough Hospital, ${ }^{5}$ Department of Laboratory Medicine and Pathobiology, ${ }^{6}$ Department of Anatomic Pathology, Sunnybrook Health Sciences Centre, and ${ }^{7}$ Departments of Medical Biophysics and Medical Imaging, University of Toronto, Toronto, Ontario, Canada

Address for correspondence: Martin J Yaffe, 2075 Bayview Avenue, S6-57, Toronto, Ontario, Canada M4N 3M5. e-mail: martin.yaffe@ sri.utoronto.ca

Keywords: breast cancer, lumpectomy, multifocality, surgical margins, whole-mount pathology 
Aims: Technical limitations in conventional pathological evaluation of breast lumpectomy specimens may reduce diagnostic accuracy in the assessment of margin and focality. A novel technique based on whole-mount serial sections enhances sampling while preserving specimen conformation and orientation. The aim of this study was to investigate assessment of focality and margin status by the use of whole-mount serial sections versus simulated conventional sections in lumpectomies.

Methods and results: Two pathologists interpreted whole-mount serial sections and simulated conventional sections for 58 lumpectomy specimens by reporting the closest margin and focality. Measurements were compared by the use of McNemar's chi-squared test. Statistically significant differences were observed in the assignment of both margin positivity $(P=0.014)$ and multifocality $(P=0.021)$. A positive margin or multifocal disease was identified by the use of whole-mount serial sections but missed in the simulated conventional assessment in $10.3 \%$ and $17.2 \%$ of all cases, respectively. There was no case in which a positive margin was detected only in the simulated conventional assessment.

Conclusions: The whole-mount technique is more sensitive than conventional assessment for identifying a positive margin or multifocal disease in breast lumpectomy specimens. Undersampling in conventional sections was implicated in almost all cases of discordance. The majority of positive margins or secondary foci identified only in wholemount serial sections concerned in-situ disease. 


\section{Introduction}

Lumpectomy surgery is part of the standard of care for T1 and T2 tumours, and offers improved cosmesis as compared with mastectomy. ${ }^{1,2}$ However, there remains a life-long risk of local recurrence. ${ }^{3,4}$ Pathological evaluation provides key descriptors of tumour extent and morphology, determines the adequacy of excision, and provides a basis for staging and planning of adjuvant therapies.

Two key descriptors are the disease status in the specimen margin and tumour focality. The prevalence of microscopic disease at the surgical margin is estimated to be $25 \% .^{5}$ A positive margin is an indication for re-excision and an important risk factor for local recurrence. ${ }^{6-9}$ Diminished local control after lumpectomy surgery increases the risk of systemic disease ${ }^{10,11}$ and is associated with reduced overall survival. ${ }^{12}$

Definitive primary surgery resulting in pathologically negative margins is more difficult to achieve when there are multiple foci of disease. Multifocality occurs in $\sim 30 \%$ of breast tumours, ${ }^{13}$ and is associated with positive margins in non-palpable tumours ${ }^{14}$ and residual disease. ${ }^{15,16}$ Older studies have associated multifocality with an increased risk of local recurrence, ${ }^{17}$ but this may reflect suboptimal surgical margin assessment. ${ }^{18,19}$ More recent studies have shown no increase in local recurrence when disease is multifocal, if all of the disease can be excised, ${ }^{20,21}$ emphasizing the importance of accurate margin assessment. Even when tumour is excised with pathologically clear margins as assessed by conventional methods, local recurrence rates are high in the absence of additional therapy. For ductal carcinoma in situ (DCIS) treated by 
lumpectomy without adjuvant radiotherapy, multifocality has been identified as a strong predictor of recurrence. $^{22}$

Technical limitations of conventional pathological assessment can give rise to inaccurate measurements, which may, in turn, increase the risk of undertreatment and recurrence. Variations in tissue handling, grossing protocols and extent of sampling of blocks for microscopic evaluation pose a challenge for pathologists, and have been associated with variable accuracy in margin evaluation. ${ }^{23-25}$ Given the impracticality of complete sampling of lumpectomies, the College of American Pathologists currently recommends microscopic examination, when practical, of the entire imaging abnormality in non-palpable lesions, and of all identified areas of gross lesion. ${ }^{26}$

Conventional techniques fail to preserve conformation and orientational context in tissues. This may further confound accurate margin estimates. ${ }^{27}$ Specimen compression has been associated with false-positive margins. ${ }^{28}$ Disagreement between disease status in the margin and in the re-excision specimen ${ }^{29}$ may reflect the interplay of conformational changes and undersampling.

Whole-mount sections provide more thorough sampling by capturing an entire cross-sectional area of the tumour, clearly showing relationships between tumour foci and margins. Whole-mount sections have demonstrated unexpected, clinically significant findings, ${ }^{30-32}$ and, for multifocal disease, can help to characterize metastatic potential on the basis of tumour distribution. ${ }^{33,34}$ Previous studies have been based on, typically, one whole-mount section, judiciously selected to represent the tumour. To enhance coverage while preserving conformation, we have incorporated serial sectioning. ${ }^{35,36}$ Here, we 
investigate estimates of margin positivity and tumour focality obtained with whole-mount serial sectioning (WMSS) versus simulated conventional sampling (SCS) from the same cases.

\section{Materials and methods}

SPECIMEN COLLECTION AND PROCESSING

The study was conducted on 58 lumpectomy specimens obtained from the Department of Anatomic Pathology at Sunnybrook Health Sciences Centre. Investigations were performed under the approval of the institutional Research Ethics Board (study ID 1922009), which waived the requirement for obtaining patient consent, and included consecutive surgeries performed from November 2009 to November 2011 with biopsyconfirmed carcinoma (of any histological type).

Specimens from 58 patients, out of a total 90 patients who were assessed for eligibility, were included in the study. Patients who received preoperative radiation or chemotherapy, or specimens that would have been sampled in toto at the time of gross specimen preparation $(n=13 ; n=9)$ or for which intraoperative consultation was required $(n=2)$, were excluded. Seven cases were excluded because of workflow constraints, and one because of cancellation of surgery post-inclusion.

Specimen preparation was performed as follows. Each specimen was oriented and inked according to standard clinical protocols. The specimen was then suspended in a buoyant, density-matched agar gel to preserve the conformation. Together with a custom tissue slicing mould, this allowed serial slicing at uniform, 4-mm intervals from the 
medial to the lateral aspect. After overnight fixation in $10 \%$ neutral buffered formalin, high-resolution optical images were acquired from the fixed tissue slices by use of a camera equipped with a telecentric lens to reduce magnification variations over the depth of field.

The fixed tissue slices were then processed by use of a custom, 16-h schedule with automatic microwave assistance (Pathos Delta; Milestone, Sorisole, Italy) to compensate for the additional time required to process large slices. Commercially available oversized cassettes (dimensions: $75 \times 52 \times 17 \mathrm{~mm}^{3}$ ) (Super Cassettes; Leica Biosystems, Wetzlar, Germany) accommodated 'smaller' whole-mount slices, and nylon bags were created to envelop the larger slices (slice area: up to $130 \times 80 \mathrm{~mm}^{2}$ ). Following paraffin embedding, one 4- $\mu$ m-thick tissue section was cut from the top of each block,

with a sledge microtome (SM 2500; Leica Microsystems, Wetzlar, Germany), or a standard rotary microtome for the 'smaller' slices. Haematoxylin and eosin staining was performed in an automatic stainer ('smaller' sections) (VariStain 24-4; Thermo Fisher Scientific, Waltham, MA, USA) or manually ('larger' sections) with custom racks and containers. $^{37}$

SCS

Because conventional pathological sampling as performed in the clinical setting would compromise tissue integrity and preclude WMSS, a strategy was devised to simulate the set of conventional histological sections that would have been obtained from each case. 
Using the optical images together with the fixed tissue slices, the pathologist assistant performed the simulation of conventional sampling as follows (Figure 1). With the cursor in place of a scalpel, areas in the optical images corresponding to the locations where a standard tissue block would be excised in the clinical setting were digitally outlined (Figure 1a). Guidance from the standard aids (patient record, palpation, and specimen slice radiography) was available as needed.

\section{IMAGE PROCESSING}

To create the WMSS image set (e.g. Figure 1b), slides were digitized with a large-area confocal scanner (Tissue Scope; Huron Technologies, Waterloo, Ontario, Canada) at $2 \mu \mathrm{m} /$ pixel resolution, which has been deemed in previous work to be adequate for tumour detection. ${ }^{38}$ To create the SCS images, each whole-mount image was overlaid with the corresponding optical image by the use of purpose-built viewing software (Sedeen Viewer; Pathcore, Toronto, Ontario, Canada). The pair of images was manually registered by the use of linear transformation parameters (translation, rotation, and scaling). The digital outlines or contours created by the pathologist assistant were transposed from the optical image to the whole-mount image, and the enclosed areas were digitally cropped from the whole-mount images to create the set of SCS images for each case (Figure 1c).

Specimen grossing diagrams were also created to assist the pathologist with orienting the SCS images. Diagrams were created in a way that simulated the practice of pathologists at our institution at the time of the study (i.e. photocopies of specimen slices), 
by applying the following algorithm to the high-resolution optical images: (i) conversion to greyscale (with the 'imadjust' function); (ii) contrast enhancement to mimic the overexposed characteristic of the generic photocopy; and (iii) application of a 'blurring' function (created with the 'fspecial' averaging filter and application of a 2D fast Fourier transform) (MATLAB R2011b; MathWorks, Natick, MA, USA).

PATHOLOGIST INTERPRETATION

Interpretation was completed between two pathologists (K.L. and J.Z.) for all 58 cases digitally on a computer screen with measurement tools in the Sedeen viewing program. A minimum 6-week 'washout period' separated the WMSS and SCS interpretation sessions for each case, and, for the SCS session, the macroscopic description and the specimen diagram were available. Tumour areas detected in each image were digitally contoured, for invasive and in-situ disease separately. The distance of closest approach of tumour to margin ink was measured with the ruler tool in Sedeen, and the number of distinct tumour foci was identified.

In the absence of standardized guidelines defining thresholds for multifocality, tumours were classified as either unifocal or multifocal as follows. Foci of either invasive disease or DCIS measured as being $>4 \mathrm{~mm}$ apart in the plane of the image (anteriorposterior and superior-inferior) were considered to be distinct. In the direction of the serial slicing (medial-lateral dimension), foci in adjacent 4-mm sections were considered to be distinct if tumour discontinuity was evident to the pathologist using the image overlay and transparency functions available in Sedeen. For non-adjacent sections, the 
tumour was always considered to be multifocal if there was at least one intervening section with no tumour cells at the corresponding location.

STATISTICAL ANALYSIS

A set of 15 lumpectomies from the WMSS image set was randomly selected to estimate inter-rater variability with intra-class correlation (ICC) analysis. The total tumour area for each specimen was computed (for invasive and in-situ disease separately) by summation of the areas enclosed in the digital contours. To provide a context of tumour coverage, summary statistics for tumour area for each presentation were computed, and the median values were compared by use of a paired $t$-test, or a Wilcoxon signed-rank test if transformed datasets failed the Shapiro-Wilk normality test. Coverage was also represented by summary statistics computed for the number of slides generated for each presentation.

The status of invasive and in-situ disease in the margin was dichotomized as either positive or negative, with a threshold of $0.1 \mathrm{~mm}$. This threshold was selected as a practical implementation of current guidelines defining a negative margin as 'no ink on tumour, ${ }^{39}$ considering potential measurement error introduced by histological artefacts and estimated imprecision in the placement of the digital measurement tool in Sedeen. Focality was dichotomized as either unifocal, or multifocal if the pathologist judged that there were two or more distinct foci. Statistical analysis for margin status and focality was performed with McNemar' s chi-squared test. 


\section{Results}

Very good reproducibility was observed for measurement of closest approach of tumour to margin ink (ICC $0.975,95 \%$ confidence interval 0.929-0.991), and absolute agreement for both dichotomized outcomes (margin status and focality). Therefore, we have pooled evaluations from the two pathologists. The results for margin and focality assessments comparing WMSS and SCS are summarized in Table 1, and indicate significant differences for both. The degrees of coverage achieved by the two presentations are compared in Table 2, and indicate highly significant differences in the mean tumour area (invasive and in situ) between the two presentations.

The proportion of all cases in which a positive margin was identified with both presentations was $6.9 \%$ (4/58). For an additional $10.3 \%$ of all lumpectomies $(6 / 58)$, a positive margin was detected only with WMSS, indicating that the sensitivity of WMSS is more than twice that of SCS for detecting a positive margin. In all six cases, SCS failed to sample the closest margin, which was composed of invasive ductal carcinoma in one case and DCIS in four cases. In the remaining, discordant case, the sampling template failed to capture the margin ink as intended, owing to a technical limitation (margin ink partially obscured or 'blurred' in the optical image because of opacity of the gel over the thickness of the slice). There were no discordant cases in which a positive margin was detected only with SCS. A key example of a positive margin that was not identified with SCS but that was apparent in the whole-mount presentation is shown in Figure 2.

WMSS is almost three times as sensitive as SCS for detecting multifocal disease. In $6.9 \%$ of all cases (4/58), multfocality was identified with both strategies. However, in 
$17.2 \%$ of all cases (10/58), multifocality was identified only in the WMSS evaluation. For these 'misses', undersampling with SCS was usually implicated (9/10), and the focus that was missed was usually DCIS (7/10). In the remaining three of 10 cases, pathologist error in interpreting the WMSS images was deemed to be responsible for the discordance, and was resolved by consensus. An example of a second focus that was not identified with SCS but was apparent in the WMSS presentation is shown in Figure 3.

When tumour area was taken as a metric for the clinically important tissue area available for examination in each presentation, coverage was significantly reduced with SCS (Table 2), especially for DCIS. Less than half of the area of DCIS obtained with WMSS (42.8\%) was available to the pathologist for SCS assessment. The median number of slides assessed with WMSS was reduced by $42.9 \%$ as compared with SCS.

\section{Discussion}

WMSS is more sensitive than SCS for detecting positive margins and multifocality in lumpectomy specimens. On the basis of current practice guidelines and relying on conventional diagnostic techniques alone, $10.3 \%$ of the patients enrolled in this study would be at risk for residual disease and not receive the necessary re-excision treatment. The results show a tendency for SCS to miss secondary foci. This may occur if the second focus or intervening normal tissue is not sampled. Alternatively, a secondary focus apparent with SCS may be missed with WMSS if a path of intervening normal tissue appears (artefactually) to be discontinuous. 
To our knowledge, this is the first technical validation study for WMSS in lumpectomy specimens, and the first in which both assessments [whole-mount and (simulated) conventional] have been performed on the same tissues. Previous studies comparing margin status used a directed approach to select a limited number of wholemount sections, or combined a whole-mount section with conventional sampling, without gel suspension. This key difference in study design might, in part, explain our markedly higher 'miss' rate for positive margins in (simulated) conventional sampling than published values. Foster et al. reported a 'miss' rate of 4\%, ${ }^{30}$ and Tucker et al. reported superior detection of positive margins with conventional sections. ${ }^{40}$ Secondary methodological differences involve specimen selection criteria and thresholds for margin positivity.

We also report significantly higher inter-rater agreement than that in the only other analysis (to our knowledge) performed with whole-mount sections. ${ }^{41}$ In addition to the key methodological difference described above for validation studies, Cserni et al. utilized a study set of cases that had more 'challenging' pathology (multifocal tumours and those with significant intraductal components), whereas the random selection of cases in our ICC analysis resulted only in tumours that were deemed to be unifocal by both pathologists. In their work, Cserni et al. also adopted more stringent criteria for multifocality, and the raters comprised a panel of seven pathologists. However, in our study, both pathologists had the benefit of access to adjacent whole-mount sections in the serial-section presentation. 
Cost-effectiveness or efficiency in whole-mount sectioning relative to conventional sampling has been demonstrated for 'large' specimens, for which large numbers of conventional slides would otherwise be required. ${ }^{31,42,43}$ In our study incorporating serial sectioning, additional technical costs were incurred. These costs were partly offset by savings, because the sampling task is eliminated, and because pathologist interpretation time is reduced by $\sim 10 \%$ as compared with diagnosis based on smallformat slides.

If costs limit the widespread adoption of WMSS in routine clinical practice, it might be most effective to concentrate implementation on DCIS, lobular carcinoma, or tumours with diffuse patterns that insinuate in tissues. Observed 'misses' with SCS are accentuated in the presence of DCIS, possibly because of the absence of gross abnormality, which increases the risk of undersampling. In the present study, the limited sample size precludes analysis of the observed effects by tumour subtype to derive statistically meaningful conclusions. ${ }^{44}$ There is consensus, however, that whole-mount sections, even at the rate of one per specimen, preferentially enhance the detection and presentation of DCIS as compared with invasive tumour. ${ }^{30,45}$ Likewise, whole-mount sections are well suited for the diagnostic challenge associated with detection of residual tumour after neoadjuvant chemotherapy. ${ }^{31}$ For adoption of WMSS into broader clinical practice, validation of immunohistochemistry should follow the guidelines established by the College of American Pathologists/American Society of Clinical Oncologists. ${ }^{46-48}$

There are two key differences between the WMSS and SCS image sets: with WMSS, conformation and spatial context are better preserved, owing to inherent 
technical differences, and tissue coverage is increased. Both effects may be responsible for findings made only with WMSS, where, in contrast to SCS, each section demonstrates four margins (anterior-posterior and superior-inferior) simultaneously at a more precisely specified distance in the mediolateral dimension. Again, the sample size is too small to allow quantification of the relative contribution of each effect to the differences in margin and focality assessment. ${ }^{44}$ However, because of the inherent bias in the study design, in which simulated conventional samples are created by digitally sampling wholemount slices (see 'Strengths and weaknesses'), coverage effects may contribute disproportionately. Both WMSS and SCS presentations benefit from specimen preparation techniques designed to preserve conformation and precisely label orientation in the mediolateral dimension. However, with SCS, the pathologist must still mentally reassemble conventional sections for each slice and draw inferences about the intervening tissues.

STRENGTHS AND WEAKNESSES

The SCS design is inherently biased in favour of SCS. This design, in which each patient serves as his or her own control, provides statistically significant technical validation results for WMSS with limited sample size. SCS is performed on tissues that are prepared and processed with WMSS techniques, and the sampling may therefore benefit from WMSS optimizations. First, tissue slices are uniformly $4 \mathrm{~mm}$ in thickness, in contrast to the variable thickness within and between slices (typically $5-15 \mathrm{~mm}$ ) in freehand tissue slicing performed in the clinical setting. The quality of the WMSS slices can facilitate 
gross identification of suspicious areas for sampling by increasing the exposed surface area of tissues. Second, the tissue-processing schedule has been optimized to minimize linear shrinkage, which is estimated to be $<9 \% .{ }^{36}$ Finally, although the optical images used to generate the specimen diagrams were degraded to simulate image quality in the photocopy used in clinical practice, the diagrams available to the pathologist for SCS interpretation inherently preserve a one-to-one correspondence between location on the diagram and that on the section. On the photocopied diagram, the estimated locations corresponding to sampling are marked freehand. With the diagrams used in SCS, the pathologist might therefore be better able to estimate orientation and distances between sections, thereby obtaining better estimates of focality than what could normally be achieved with real sampling.

The bias in favour of SCS may be offset by the ability of the pathologist to add blocks at his or her discretion in real practice. Blocks targeting the corresponding intervening tissue may be added when the initial set of slides reveal cancer in nonsequential slices. In the current study, simulation of conventional sampling was limited to one session.

We have demonstrated clinically significant discordances in margin and focality measurements between WMSS and SCS, with the tendency for SCS to fail to identify positive margins or multifocal disease. WMSS may offer the greatest clinical advantage when used in the assessment of DCIS and tumours with an extensive intraductal component with narrow negative margins. It remains to be demonstrated whether the recurrence risk is lowered when treatment decisions are based on WMSS assessment. 


\section{Conflicts of interest}

None of the authors has a competing interest to declare.

\section{Author contributions}

Conception and design: C. M. B. Holloway, G. M. Clarke, J. T. Zubovits, and M. J. Yaffe. Financial support: G. M. Clarke and M. J. Yaffe. Provision of study materials or patients: C. M. B. Holloway, J. T. Zubovits, and S. Nofech-Mozes. Collection and assembly of data: D. Wang, G. M. Clarke, K. Liu, and M. Murray. Pathological evaluation: J. T. Zubovits and K. Liu. Data analysis and interpretation: C. M. B. Holloway, D. Wang, G. M. Clarke, J. T. Zubovits, S. Nofech-Mozes, and M. J. Yaffe. Manuscript writing, figure preparation, and review: all authors. Final approval of manuscript: all authors.

\section{Acknowledgements}

This study was funded by the Canadian Breast Cancer Research Alliance/Canadian Cancer Society Research Institute. The authors are grateful to Ms Anoma Gunasekara for her assistance with recruitment, and together with Ms Yulia Yerofeyeva, with organizing all aspects of the study logistics and patient tracking. We also thank Dr Alex Kiss for statistical consultation, from study design to interpretation of results. Finally, we acknowledge the cooperation of pathologist assistants Ms Anna-Marie Moskaluk, Mr Ian 
Cooper, Dr Laibao Sun and Dr Peter Leventis in performing the virtual sampling and overseeing the research protocol, and Adebayo Adeeko for assistance with specimen preparation. 


\section{References}

1. Kramer S, Darsow M, Kummel S, Kimmig R, Rezai M. Breast-conserving treatment of breast cancer-oncological and reconstructive aspects. Gynakol. Geburtshilfliche Rundsch. 2008; 48; 56-62.

2. Schwartz GF, Veronesi U, Clough KB et al. Consensus conference on breast conservation. J. Am. Coll. Surg. 2006; 203; 198-207.

3. Komoike Y, Akiyama F, lino Y et al. Ipsilateral breast tumor recurrence (IBTR) after breast-conserving treatment for early breast cancer: risk factors and impact on distant metastases. Cancer 2006; 106; 35-41.

4. Kreike B, Hart AAM, van de Velde T et al. Continuing risk of ipsilateral breast relapse after breast-conserving therapy at long-term follow-up. Int. J. Radiat. Oncol. Biol. Phys. 2008; 71; 1014-1021.

5. McCahill LE, Single RM, Aiello Bowles EJ et al. Variability in reexcision following breast conservation surgery. JAMA 2012; 307; 467-475.

6. van Dongen JA, Voogd AC, Fentiman IS et al. Long-term results of a randomized trial comparing breast-conserving therapy with mastectomy: European Organization for Research and Treatment of Cancer 10801 trial. J. Natl Cancer Inst. 2000; 92; 1143-1150.

7. Park CC, Mitsumori M, Nixon A et al. Outcome at 8 years after breast-conserving surgery and radiation therapy for invasive breast cancer: influence of margin status and systemic therapy on local recurrence. J. Clin. Oncol. 2000; 18; 1668-1675. 
8. Singletary SE. Surgical margins in patients with early-stage breast cancer treated with breast conservation therapy. Am. J. Surg. 2002; 184; 383-393.

9. Luini A, Rososchansky J, Gatti G et al. The surgical margin status after breastconserving surgery: discussion of an open issue. Breast Cancer Res. Treat. 2009; $113 ; 397-402$.

10. Kingsmore D, Hole D, Gillis C. Why does specialist treatment of breast cancer improve survival? The role of surgical management. Br. J. Cancer 2004; 90; $1920-1925$.

11. Punglia RS, Morrow M, Winer EP, Harris JR. Local therapy and survival in breast cancer. N. Engl. J. Med. 2007; 356; 2399-2405.

12. Clarke M, Collins R, Darby S et al. Effects of radiotherapy and of differences in the extent of surgery for early breast cancer on local recurrence and 15-year survival: an overview of the randomised trials. Lancet 2005; 366; 2087-2106.

13. Holland R, Veling SH, Mravunac M, Hendriks JH. Histologic multifocality of Tis, T1-2 breast carcinomas. Implications for clinical trials of breast-conserving surgery. Cancer 1985; 56; 979-990.

14. Reedijk M, Hodgson N, Gohla G et al. A prospective study of tumor and technical factors associated with positive margins in breast-conservation therapy for nonpalpable malignancy. Am. J. Surg. 2012; 204; 263-268.

15. Vaidya JS, Vyas JJ, Chinoy RF, Merchant N, Sharma OP, Mittra I. Multicentricity of breast cancer: whole-organ analysis and clinical implications. Br. J. Cancer 1996; 74; 820-824. 
16. Zavagno G, Goldin E, Mencarelli R et al. Role of resection margins in patients treated with breast conservation surgery. Cancer 2008; 112; 1923-1931.

17. Leopold KA, Recht A, Schnitt SJ et al. Results of conservative surgery and radiation therapy for multiple synchronous cancers of one breast. Int. J. Radiat. Oncol. Biol. Phys. 1989; 16; 11-16.

18. Kurtz JM, Jacquemier J, Amalric R et al. Breast-conserving therapy for macroscopically multiple cancers. Ann. Surg. 1990; 212; 38-44.

19. Wilson LD, Beinfield M, McKhann CF, Haffty BG. Conservative surgery and radiation in the treatment of synchronous ipsilateral breast cancers. Cancer 1993; 72; 137-142.

20. Gentilini O, Botteri E, Rotmensz N et al. Conservative surgery in patients with multifocal/multicentric breast cancer. Breast Cancer Res. Treat. 2009; 113; 577583.

21. Haley MM, Garces YI, Kee AY et al. Breast conservation therapy (BCT) in multifocal vs. unifocal early stage breast cancer patients: the Mayo Experience. Int. J. Radiat. Oncol. Biol. Phys. 2007; 69; S208-S209.

22. Rakovitch E, Nofech-Mozes S, Hanna W et al. A population-based validation study of the DCIS Score predicting recurrence risk in individuals treated by breastconserving surgery alone. Breast Cancer Res. Treat. 2015; 152; 389-398.

23. Wright MJ, Park J, Fey JV et al. Perpendicular inked versus tangential shaved margins in breast-conserving surgery: does the method matter? J. Am. Coll. Surg. 2007; 204; 541-549. 
24. Gibson GR, Lesnikoski BA, Yoo J, Mott LA, Cady B, Barth RJ. A comparison of ink-directed and traditional whole-cavity re-excision for breast lumpectomy specimens with positive margins. Ann. Surg. Oncol. 2001; 8; 693-704.

25. Dillon MF, Hill ADK, Quinn CM, McDermott EW, O’Higgins N. A pathologic assessment of adequate margin status in breast-conserving therapy. Ann. Surg. Oncol. 2006; 13; 333-339.

26. Lester S, Bose S, Chen Y-Y, Connolly JL, de Baca M. Protocol for the examination of specimens from patients with invasive carcinoma of the breast protocol applies to all invasive carcinomas of the breast, including ductal. Northfield, IL, USA: College of American Pathologists; 2013. Available at: http://www.cap.org/ShowProperty?nodePath=/UCMCon/Contribution\%20Folders/ WebContent/pdf/cp-breast-invasive-13protocol-3200.pdf

27. Graham RA., Homer MJ, Katz J, Rothschild J, Safaii H, Supran S. The pancake phenomenon contributes to the inaccuracy of margin assessment in patients with breast cancer. Am. J. Surg. 2002; 184; 89-93.

28. Dooley WC, Parker J. Understanding the mechanisms creating false positive lumpectomy margins. Am. J. Surg. 2005; 190; 606-608.

29. Neuschatz AC, DiPetrillo T, Steinhoff M et al. The value of breast lumpectomy margin assessment as a predictor of residual tumor burden in ductal carcinoma in situ of the breast. Cancer 2002; 94; 1917-1924.

30. Foster MR, Harris L, Biesemier KW. Large format histology may aid in the detection of unsuspected pathologic findings of potential clinical significance: a 
prospective multiyear single institution study. Int. J. Breast Cancer 2012; 2012; 532547.

31. Ibarra JA. The value of combined large format histopathology technique to assess the surgically removed breast tissue following neoadjuvant chemotherapy: a single institution study of 40 cases. Int. J. Breast. Cancer 2012; 2012; 361707.

32. Foschini MP, Baldovini C, Ishikawa Y, Eusebi V. The value of large sections in surgical pathology. Int. J. Breast Cancer 2012; 2012; 785947.

33. Tot T, Pekar G, Hofmeyer S, Sollie T, Gere M, Tarjan M. The distribution of lesions in $1-14-\mathrm{mm}$ invasive breast carcinomas and its relation to metastatic potential. Virchows Arch. 2009; 455; 109-115.

34. Tot $\mathrm{T}$. Clinical relevance of the distribution of the lesions in 500 consecutive breast cancer cases documented in large-format histologic sections. Cancer 2007; $110 ; 2551-2560$.

35. Clarke GM, Murray M, Holloway CMB, Liu K, Zubovits JT, Yaffe MJ. 3D pathology volumetric technique: a method for calculating breast tumour volume from whole-mount serial section images. Int. J. Breast Cancer 2012; 2012; 691205.

36. Sun L, Wang D, Zubovits JT, Yaffe MJ, Clarke GM. An improved processing method for breast whole-mount serial sections for three-dimensional histopathology imaging. Am. J. Clin. Pathol. 2009; 131; 383-392.

37. Clarke GM, Eidt S, Sun L, Mawdsley G, Zubovits JT, Yaffe MJ. Whole-specimen histopathology: a method to produce whole-mount breast serial sections for 3-D digital histopathology imaging. Histopathology 2007; 50; 232-242. 
38. Clarke GM, Zubovits JT, Katic M, Peressotti C, Yaffe MJ. Spatial resolution requirements for acquisition of the virtual screening slide for digital wholespecimen breast histopathology. Hum. Pathol. 2007; 38; 1764-1771.

39. Moran MS, Schnitt SJ, Giuliano AE et al. Society of Surgical Oncology-American Society for Radiation Oncology consensus guideline on margins for breastconserving surgery with whole-breast irradiation in stages I and II invasive breast cancer. Ann. Surg. Oncol. 2014; 21; 704-716.

40. Tucker FL. Imaging-assisted large-format breast pathology: program rationale and development in a nonprofit health system in the United States. Int. J. Breast Cancer 2012; 2012; 171792.

41. Cserni G, Bori R, Sejben I et al. Unifocal, multifocal and diffuse carcinomas: a reproducibility study of breast cancer distribution. Breast 2013; 22; 34-38.

42. Méchine-Neuville A, Chenard MP, Gairard B, Mathelin C, Bellocq JP. [Large sections in routine breast pathology. A technique adapted to conservative surgery]. Ann. Pathol. 2000; 20; 275-279.

43. Tot T. Cost-benefit analysis of using large-format histology sections in routine diagnostic breast care. Breast 2010; 19; 284-288.

44. Harrell F. Regression modeling strategies. New York: Springer-Verlag, 2001.

45. Jackson PA, Merchant W, McCormick CJ, Cook MG. A comparison of large block macrosectioning and conventional techniques in breast pathology. Virchows Arch. 1994; 425; 243-248.

46. Fitzgibbons PL, Murphy DA, Hammond MEH, Allred DC, Valenstein PN. 
Recommendations for validating estrogen and progesterone receptor immunohistochemistry assays. Arch. Pathol. Lab. Med. 2010; 134: 930-935.

47. Wolff AC, Hammond ME, Hicks DG et al. Recommendations for human epidermal growth factor receptor 2 testing in breast cancer: American Society of Clinical Oncology/College of American Pathologists Clinical Practice Guideline Update. CAP/ASCO Guidel. Data Suppl. 2014. Available at: http://www.cap.org/ShowProperty?nodePath=/UCMCon/Contribution Folders/WebContent/pdf/her2-data-supplement.pdf (accessed 27 October 2015).

48. Wolff AC, Hammond MEH, Hicks DG et al. Recommendations for human epidermal growth factor receptor 2 testing in breast cancer: American Society of Clinical Oncology/College of American Pathologists clinical practice guideline update. Arch. Pathol. Lab. Med. 2014; 138; 241-256. 
Figure 1. Strategy for generating simulated conventional sections. a, Optical image of a representative formalin-fixed tissue slice. Areas selected for sampling by the pathologist assistant, on the basis of gross evaluation, were indicated digitally by the placement of cassette-size cyan boxes. b, Corresponding whole-mount section created from the slice in (a) (haematoxylin and eosin). Cyan boxes from (a) are overlaid to highlight the regions that were selected by the pathologist assistant. c, Simulated conventional sections imitating the slides that would be available to the pathologist through conventional sampling, generated by cropping the selected areas from the image in (b). The simulation ensures that the evaluating pathologist is blinded to findings outside the prescribed areas, and needs to map relevant findings back to the grossing diagram.

Figure 2. Example of margin discordance. a, Optical image of a formalin-fixed tissue slice. The tissue is predominantly fatty, with no obvious mass-forming lesion. No region from this slice was selected by the pathologist assistant for simulated conventional sampling. b, A focus of invasive lobular carcinoma (green contour) was identified on a whole-mount section. The orange contour highlights the location of the positive margin. A region enclosing the positive margin is contoured by the black rectangle. c, Positive margin from an enlargement of the region in the black rectangle from (b). A, anterior; I, inferior; P, posterior; S, superior.

Figure 3. Invasive ductal carcinoma not otherwise specified, with a separate focus of ductal carcinoma in situ (DCIS) that was detected only in the whole-mount serial 
sectioning (WMSS) presentation. A focus of DCIS is separated from the dominant mass of invasive carcinoma, and was not sampled during the simulation process. a-d, Four sequential 4-mm-thick formalin-fixed slices with simulated conventional sampling indicated by the cyan boxes. Sampling was directed towards a grossly identified mass lesion in (d) and corresponding tissue medial to the mass. $\mathbf{e}-\mathbf{h}$, The whole-mount sections corresponding to the formalin-fixed slices in (a)-(d): areas of DCIS and invasive disease identified in the WMSS are contoured in yellow and green, respectively, and are each enclosed in a black rectangle $(\mathbf{e}, \mathbf{h})$. i, Enlargement of the area enclosed in the black rectangle in (e) (DCIS). j, Enlargement of the area enclosed in the black rectangle in (h) (invasive carcinoma). A, anterior; I, inferior; P, posterior; S, superior. 
(a) $\stackrel{5 m m}{\text { m }}$

(b) ${ }^{5 m m}$

1

A

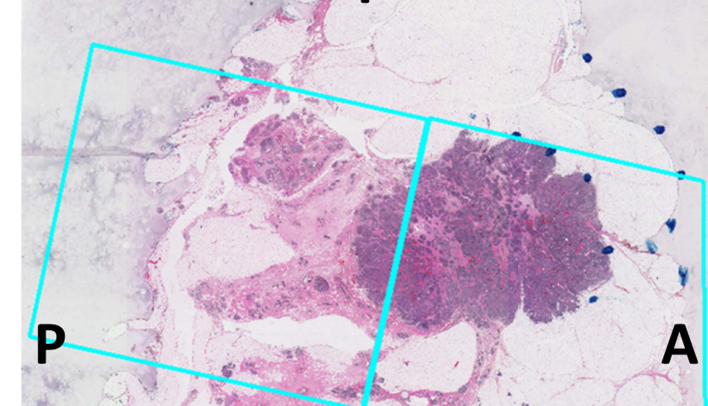

(c)

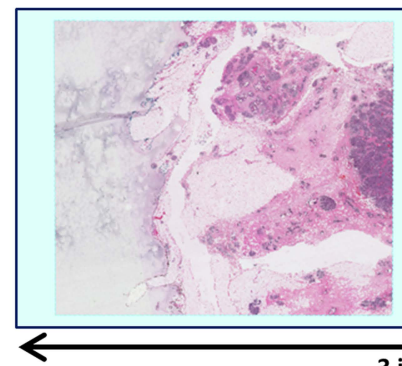

3 inches [76.2 mm]

Simulated

Conventional

Section \#1

1 inch [25.4 mm]

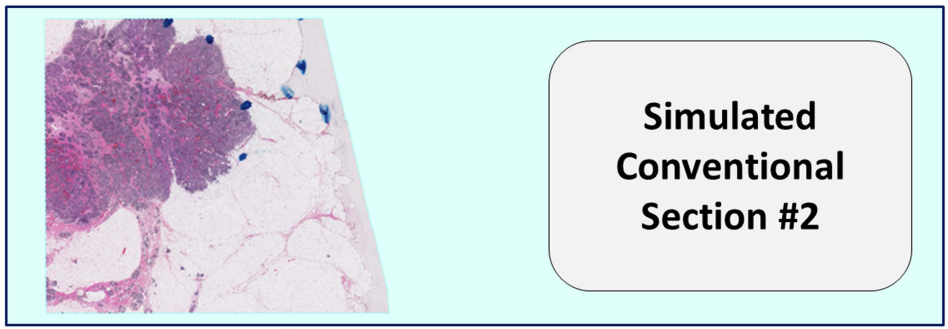

Figure 1.

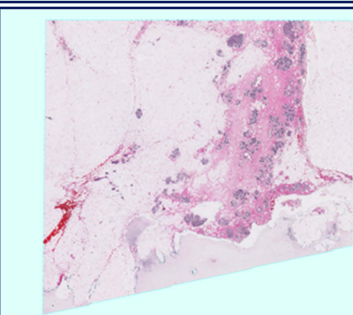

Simulated Conventional Section \#3 

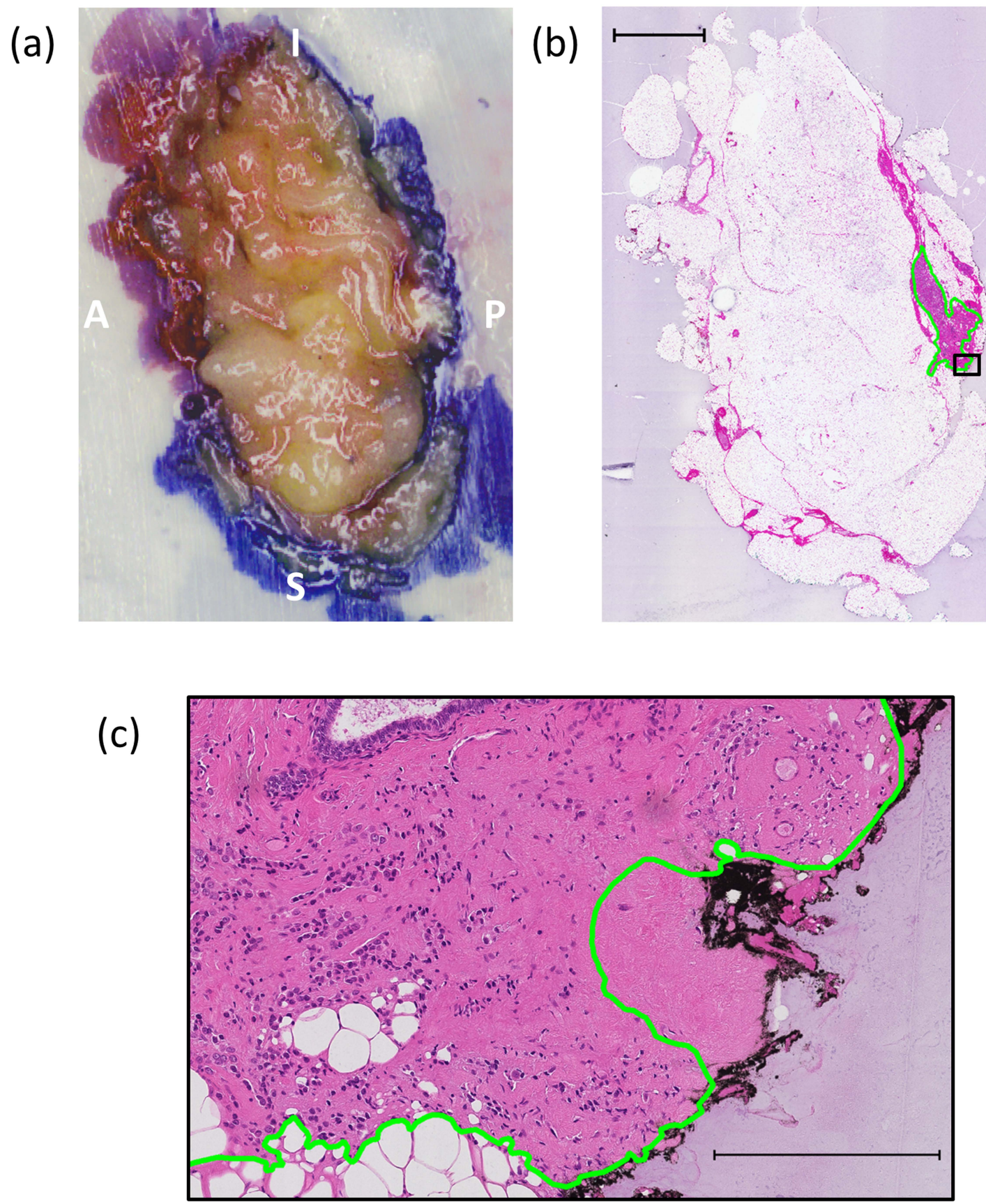

Figure 2. 
(a)

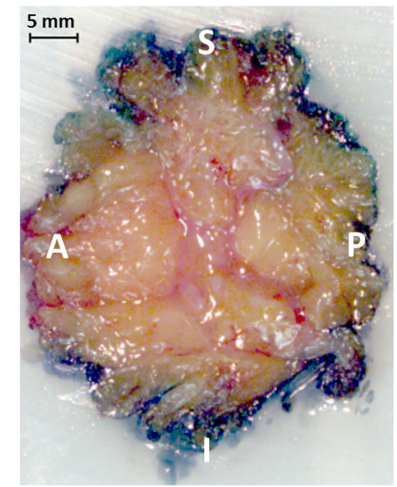

(e)
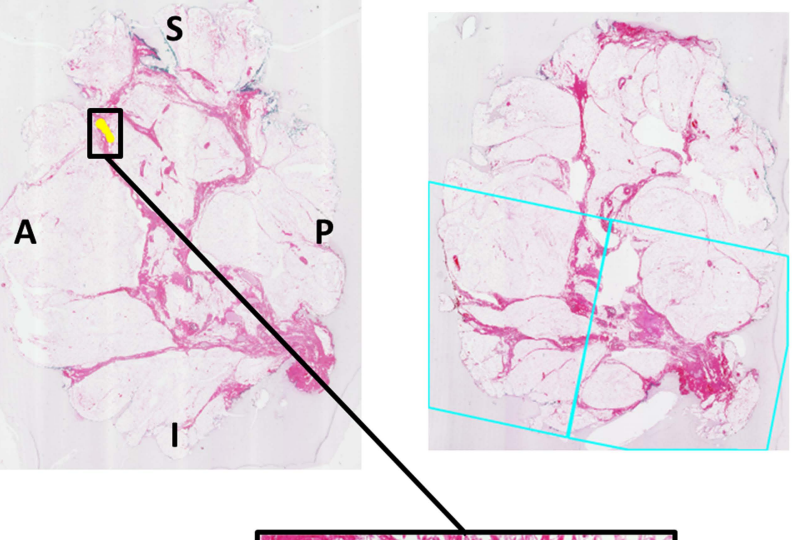

(i)
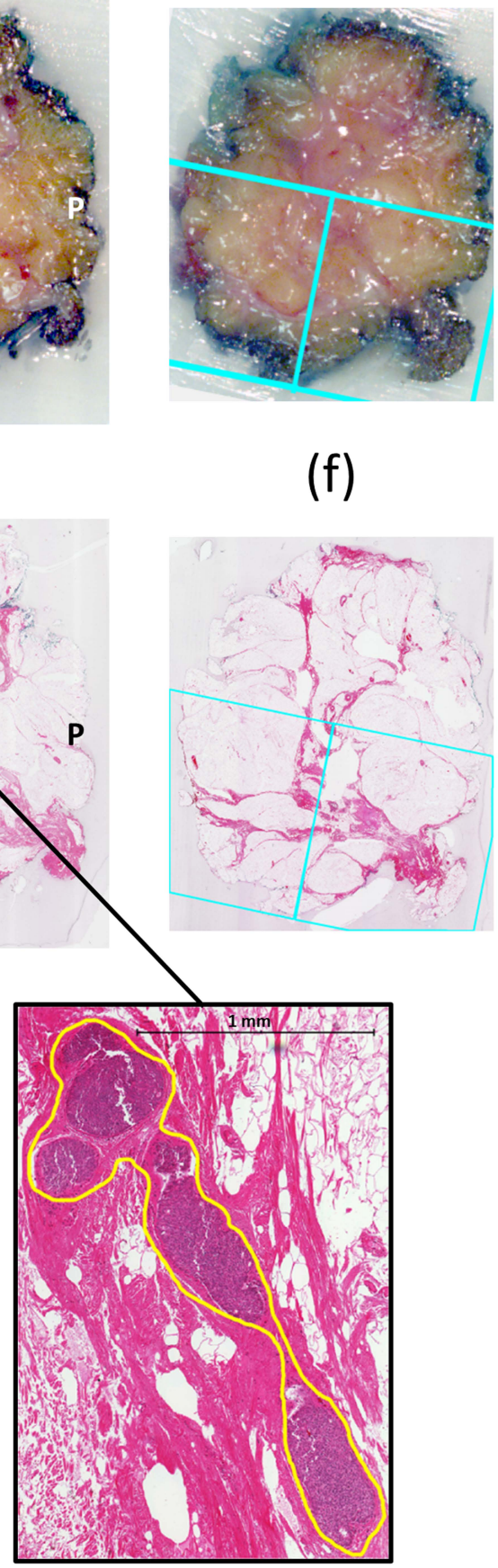

(f) (c)

(g)

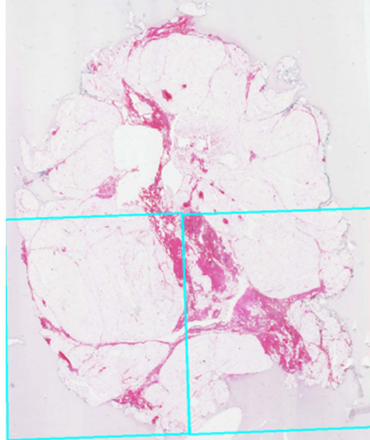

(h)
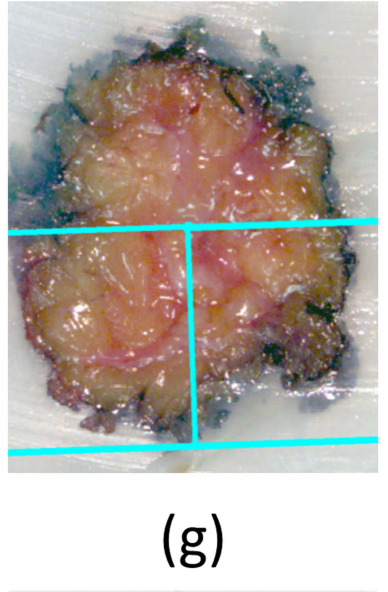

(d)
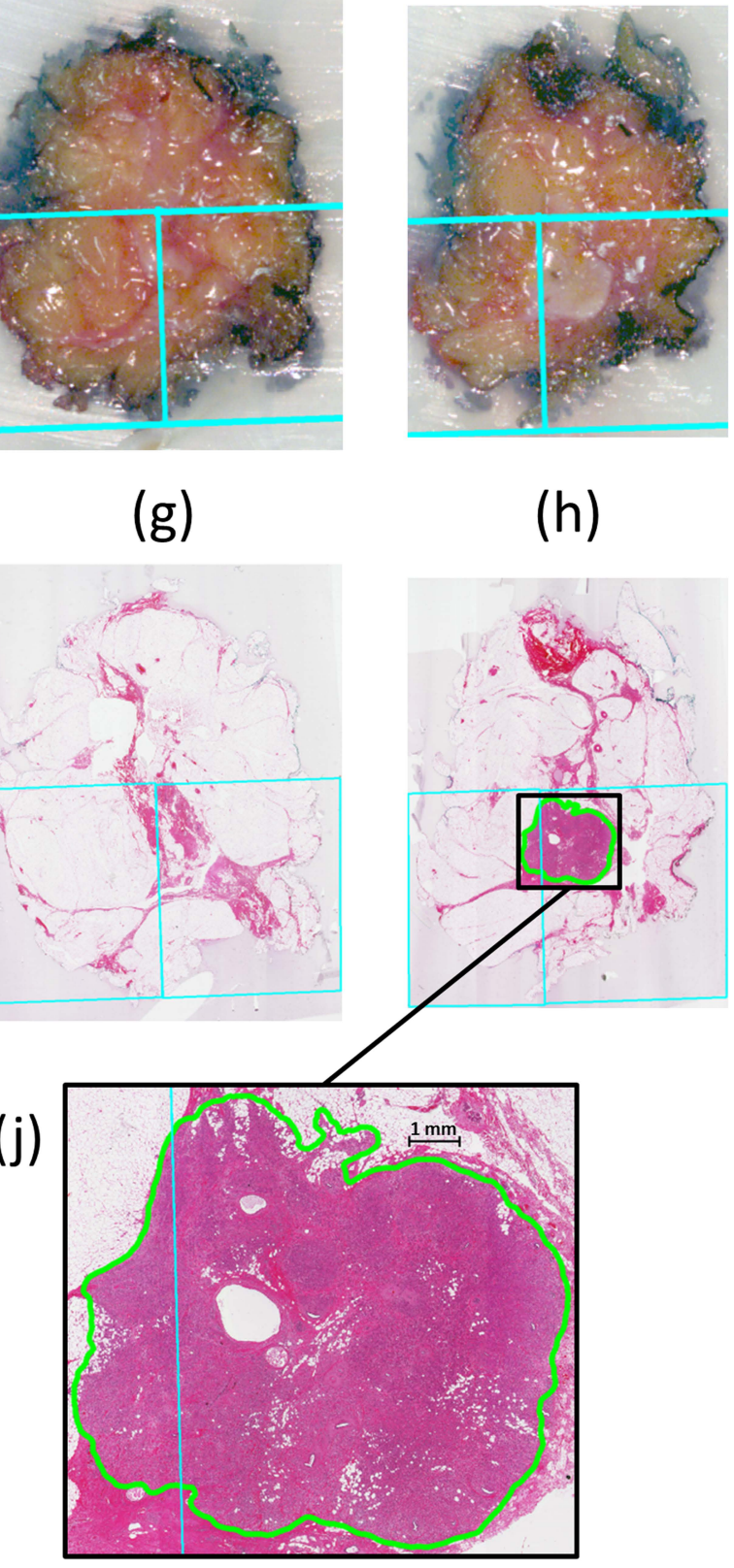

Figure 3. 
Table 1. Comparison of tumour measurements obtained from whole-mount serial sections with those from simulated conventional sections

\begin{tabular}{|c|c|c|c|c|c|c|}
\hline \multicolumn{7}{|c|}{ Whole-mount serial sections $(n=58)$} \\
\hline \multirow{4}{*}{$\begin{array}{l}\text { Simulated } \\
\text { conventional } \\
\text { sections } \\
(n=58)\end{array}$} & \multicolumn{3}{|c|}{$\begin{array}{l}\text { Margins } \\
\chi^{2}=6, P=0.014\end{array}$} & \multicolumn{3}{|c|}{$\begin{array}{l}\text { Focality } \\
\chi^{2}=5.3, P=0.021\end{array}$} \\
\hline & & + & - & & $=1$ & $>1$ \\
\hline & + & $4(6.9 \%)$ & $0(0 \%)$ & $=1$ & $\begin{array}{l}42 \\
(72.4 \%)\end{array}$ & $\begin{array}{l}10 \\
(17.2 \%)\end{array}$ \\
\hline & - & $6(10.3 \%)$ & $48(82.7 \%)$ & $>1$ & $\begin{array}{l}2 \\
(3.4 \%)\end{array}$ & $\begin{array}{l}4 \\
(6.9 \%)\end{array}$ \\
\hline
\end{tabular}

A positive margin was defined as tumour within $0.1 \mathrm{~mm}$ of the specimen ink. McNemar's chisquare $\left(\chi^{2}\right)$ values and the associated $P$-values are shown,and the percentages shown are proportionate to the 58 cases in the study. 
Table 2. Comparison of tumour coverage between whole-mount serial sections and simulated conventional sections for the 58 cases in the study

\begin{tabular}{|c|c|c|c|c|}
\hline & $\begin{array}{l}\text { Tumour area, } \\
\text { invasive }\left(\mathrm{mm}^{2}\right) \text { : } \\
\text { median (minimum, } \\
\text { maximum) }\end{array}$ & $\begin{array}{l}\text { Tumour area, } \\
\text { in situ }\left(\mathrm{mm}^{2}\right) \text { : } \\
\text { median (minimum, } \\
\text { maximum) }\end{array}$ & $\begin{array}{l}\text { Number of } \\
\text { slides: } \\
\text { median } \\
\text { (minimum, } \\
\text { maximum) }\end{array}$ & Slide size $\left(\mathrm{cm}^{2}\right)$ \\
\hline $\begin{array}{l}\text { Whole-mount } \\
\text { serial sections }\end{array}$ & $333.6(0,2511.0)$ & $3.5(0,191.7)$ & $16(12,35)$ & $\begin{array}{l}5.08 \times 7.62 \\
7.62 \times 10.16\end{array}$ \\
\hline $\begin{array}{l}\text { Simulated } \\
\text { conventional } \\
\text { sections }\end{array}$ & $290.3(0,1491.8)$ & $1.5(0,127.3)$ & $28(9,53)$ & $2.54 \times 7.62$ \\
\hline $\begin{array}{l}\% \text { of whole-mount } \\
\text { serial sections }\end{array}$ & $87.0(P \leq 0.0001)$ & $42.8(P<0.0001)$ & NA & NA \\
\hline
\end{tabular}

NA - not applicable.

Summary statistics for tumour area were computed on the basis of regions enclosed by the pathologist with digital contouring. The tumour area captured in simulated conventional sections is also represented as a proportion of the tumour area identified in whole-mount serial sections, the medians are compared, and $P$-values are shown (Wilcoxon signed-rank test). For each format, the number of slides is compared, along with the size of microscope glass utilized in each format to represent the theoretical maximum area of tissue examined. In whole-mount serial sections, the median number of slides represents approximately equal numbers of 'smaller' $\left(5.08 \times 7.62 \mathrm{~cm}^{2}\right)$ and 'larger' 
$\left(7.62 \times 1.016 \mathrm{~cm}^{2}\right)$ whole-mount slides. Median numbers of slides are rounded to the nearest integer. 